\title{
Analysis of magnetoresistance in arrays of connected nano-rings
}

\author{
Giuliano Bordignon $^{\dagger *}$, Thomas Fischbacher*, Matteo Franchin ${ }^{\dagger *}$, \\ Jurgen P. Zimmermann*, Alexander A. Zhukov ${ }^{\dagger}$, Vitali V. Metlushko ${ }^{\ddagger}$, Peter A. J. de Groot ${ }^{\dagger}$, Hans Fangohr* \\ ${ }^{*}$ School of Engineering Sciences, University of Southampton, SO17 1BJ Southampton, United Kingdom \\ ${ }^{\dagger}$ School of Physics and Astronomy, University of Southampton, SO17 1BJ Southampton, United Kingdom \\ $\ddagger$ Department of Electrical and Computer Engineering, University of Illinois at Chicago, Chicago, IL 60607-0024, USA \\ Email: fangohr@soton.ac.uk
}

\begin{abstract}
We study the Anisotropic Magneto-Resistance (AMR) of a two-dimensional periodic square array of connected permalloy rings with periodicity of $1 \mu \mathrm{m}$ combining experimental and computational techniques.

The computational model consists of two parts: (i) the computation of the magnetization and (ii) the computation of the current density. For (i), we use standard micromagnetic methods. For (ii), we start from a potential difference applied across the sample, compute the resulting electric potential and subsequently the corresponding current density based on a uniform conductivity. We take into account the backreaction of the magnetoresistive effects onto the current density by self consistently computing the current density and conductivity until they converge.

We compare the experimentally measured AMR curve (as a function of the applied field) with the numerically computed results and find good agreement. The numerical data provides insight into the characteristics of the AMR data.

Finally, we demonstrate the importance of taking into account the spatial variation of the current density when computing the AMR.
\end{abstract}

\section{INTRODUCTION}

In recent years the exploration of magnetic structures at sub-micron length scale has produced a major impact on fundamental physics and technological applications. Magnetic Random Access Memories (MRAM) are among the latest achievements of such research, and arrays of tiny magnetic elements are the most promising candidates for the next generation of data storage devices.

The control of the switching behaviour in these elements is one of the major issues to be addressed in patterned magnetic data storage. Ring structures are potential candidates for such storage media [1] and resistive effects are an important factor in their design. Magnetic properties of isolated nano-rings have been reported in several publications [2], [3], [4], and here we investigate the transport properties of a system of connected rings.

\section{THE SAMPLE}

Fig. 1(a) shows a Scanning Elecron Microscopy (SEM) image of the sample we study. Periodic square arrays of $25 \mathrm{~nm}$ thick permalloy nano-rings have been prepared by e-beam lithography on a (100) silicon wafer spin-coated with bi-layer resists (PMMA and P(MMA-MAA) copolymer). Using e-beam evaporation in a high vacuum, a permalloy film

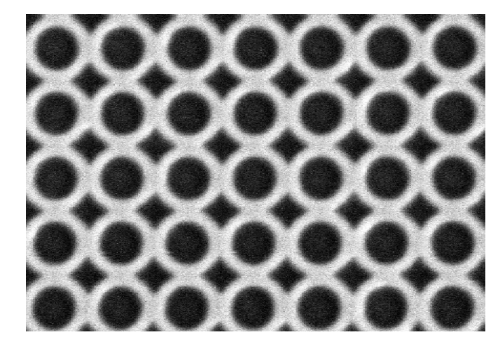

(a)

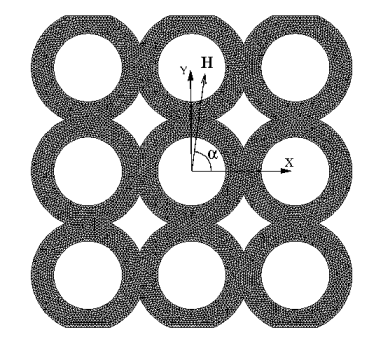

(b)
Fig. 1. (a) SEM image of the connected rings array and (b) model used in the simulations, where the field $H$ is applied nearly along the $y$-axis $\left(\alpha=88^{\circ}\right)$. The potential is applied between the top and bottom of the system, with the contact regions along the cut rings.

was deposited, and after a lift-off in acetone the arrays of magnetic permalloy rings were obtained. The distance between the centres of the rings is $1000 \mathrm{~nm}$.

\section{Computational Model}

To study the transport properties of this system we use micromagnetic simulations to find the magnetization distribution for each applied field and solve the equations describing the AMR effect via the finite element method as detailed below.

For the micromagnetic modelling we use $O O M M F$ [5] to simulate a 3 by 3 ring array as shown in Fig. 1(b). According to the experimental sample, the dimensions for the inner and outer radius are $325 \mathrm{~nm}$ and $550 \mathrm{~nm}$, respectively.

The AMR is the result of the electrons spin-orbit interaction, so that the scattering of the conduction electrons depends on the orientation of the magnetization with respect to the direction of the electric current [6]. The net effect is to have maxima in the local resistivity when the local magnetization $\mathbf{M}$ is parallel or antiparallel to the local electric current density $\mathbf{J}$. With $\theta=\angle(\mathbf{M}, \mathbf{J})$ being the angle between $\mathbf{M}$ and $\mathbf{J}$, the resistivity $\rho$ is given by [7]

$$
\rho(\theta)=\rho_{0}\left(1+\alpha \cdot \cos ^{2} \theta\right)
$$

where $\rho_{0}$ is the resistivity of the material in the absence of AMR and $\alpha$ is a coefficient that for permalloy is between $1 \%$ and $5 \%$. In our analysis we used $\rho_{0}=15 \mu \Omega / \mathrm{cm}$ [7] and $\alpha=3.5 \%$ [8]. 
To compute the AMR numerically, we compute the electric potential $\Phi$ for the system which defines the electric field $\mathbf{E}$ via

$$
\mathbf{E}=-\nabla \Phi
$$

Given $\mathbf{E}$ and a conductivity $\sigma$ (which is a function of position) we can compute the current density

$$
\mathbf{J}=\sigma \mathbf{E}=-\sigma \nabla \Phi
$$

with suitable boundary conditions.

In more detail, we need to consider the conservation of charge $\frac{\partial \rho}{\partial t}+\nabla \cdot \mathbf{J}=0$ which simplifies in the electrostatic case to

$$
\nabla \cdot \mathbf{J}=0 .
$$

We also need to impose the boundary condition, outside the contact regions, that the current must not leave the sample

$$
\hat{\mathbf{n}} \cdot \mathbf{J}=0
$$

where $\hat{\mathbf{n}}$ is a vector normal to the surface. Combining (4), (3) and (2), we obtain:

$$
\nabla \cdot \mathbf{J}=\nabla \cdot(\sigma \mathbf{E})=-\nabla \cdot(\sigma \nabla \Phi)=0 .
$$

Together with (5) and fixing the values for $\Phi$ where the electric contacts are mounted on the sample, the problem is fully defined and can be solved numerically to obtain $\Phi$ and therefore $\mathbf{E}$ and $\mathbf{J}$ for a given conductivity $\sigma$. (We do not need to consider the Lorentz force acting on the current density as the applied field is in the plane of the film.) Because the conductivity $\sigma$

$$
\sigma(\theta)=\frac{1}{\rho(\theta)}=\frac{\sigma_{0}}{1+\alpha \cdot \cos ^{2} \theta}
$$

is a function of the angle $\theta$ between magnetization $\mathbf{M}$ and current density $\mathbf{J}$, we need to solve (7) and (6) iteratively until we reach self-consistency for both the current and the conductivity distributions.

Experimentally a current density above the order of $10^{8} \mathrm{~A} / \mathrm{cm}^{2}$ affects the magnetization distribution, but the very low current density regime (of the order of $10^{3} \mathrm{~A} / \mathrm{cm}^{2}$ ) considered in our analysis makes the equilibrium magnetization independent of the applied electric field for each value of the external magnetic field. Therefore we assume the transfer of momentum between the current and the magnetization to be negligible, and the only interaction between the two fields occurs through the conductivity redistribution.

The values of the magnetization obtained from the $O O M M F$ structured grid are interpolated over a two dimensional unstructured mesh with an average edge length of $30 \mathrm{~nm}$ (Fig. 1(b)). This mesh is used to solve equations (7) and (6) to (iteratively) compute the equilibrium angle $\theta$ between $\mathbf{M}$ and J. All calculations apart from the micromagnetic modelling are performed using nmag [9], a multiphysics package based on the finite element method. (In principle, nmag is able to also do the micromagnetic simulations although at the time of writing it was not yet sufficiently fast to deal with such a large computational problem.) While we have computed magnetization data on the $3 \times 3$ ring system shown in Fig. 1(b),
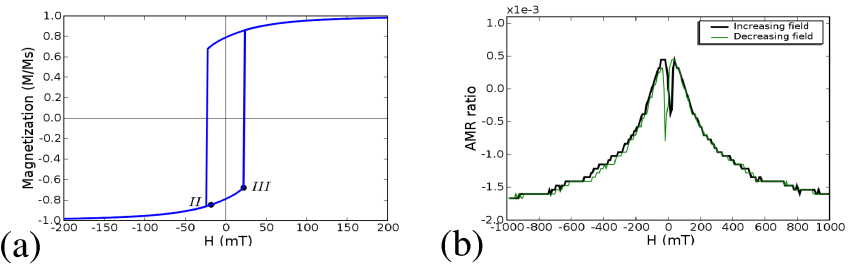

\section{(a)}

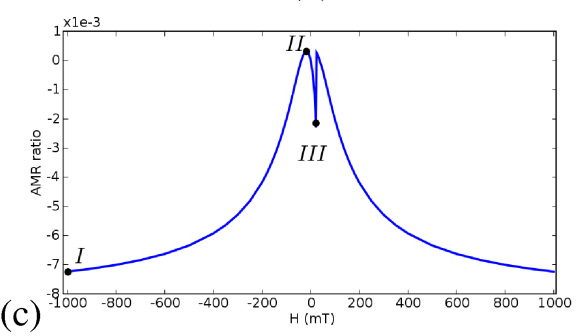

Fig. 2. (a) Simulated hysterises loop, (b) experimentally obtained AMR, (c) numerically obtained AMR. The marked points I, II and III in (c) are discussed in detail and shown in Fig. 3. Note that the experimental AMR data in (b) contains both branches (for increasing and decreasing applied magnetic field) whereas the simulated data (c) contains only AMR values for increasing field.

for our analysis of the conductivity we use only the central ring to minimise surface effects.

The AMR ratio $r_{\mathrm{AMR}}$ is then computed for each point on the hysteresis curve in terms of the resistance $R=V / I$ as

$$
r_{\mathrm{AMR}}=\frac{R(H)}{R(H=0)}-1
$$

where $V$ is the voltage between the top and bottom interfaces of the system, $I$ is the integral of the current density $\mathbf{J}$ over the contacts surface and $H$ the applied magnetic field.

\section{RESUlTS}

We study the AMR for an external magnetic field acting (nearly) along the $y$-axis (see Fig. 1(b)). The field is varied from $-1 \mathrm{~T}$ to $1 \mathrm{~T}$ in steps of $2 \mathrm{mT}$, and for each field strength the corresponding conductivity and AMR value is computed. The resulting hysteresis loop is shown in Fig. 2(a) together with the AMR data obtained experimentally (Fig. 2(b)) and numerically (Fig. 2(c)). The qualitative agreement between the measured and simulated AMR curve is good. We do not expect quantitative agreement as our model uses a small number of rings compared to the real sample and, although we restrict the analysis to the central ring, surface effects may still play a relevant role on the magnetization distribution.

We will now discuss the origins of the observed AMR data for the points labelled I, II and III in Fig. 2(c). Fig. 3 shows the magnetization and current distribution (left) and the resulting conductivity (right) for each of the points I, II and III. From Eq. (7) the conductivity is largest when the current density and the magnetization are orthogonal to each other, and smallest when they are parallel.

At point I, the applied field of $1000 \mathrm{mT}$ is fairly strong and the magnetization is mostly aligned with the negative $y$-axis. The current density needs to follow the shape of the sample and is slightly larger at the inner boundary of each ring. 


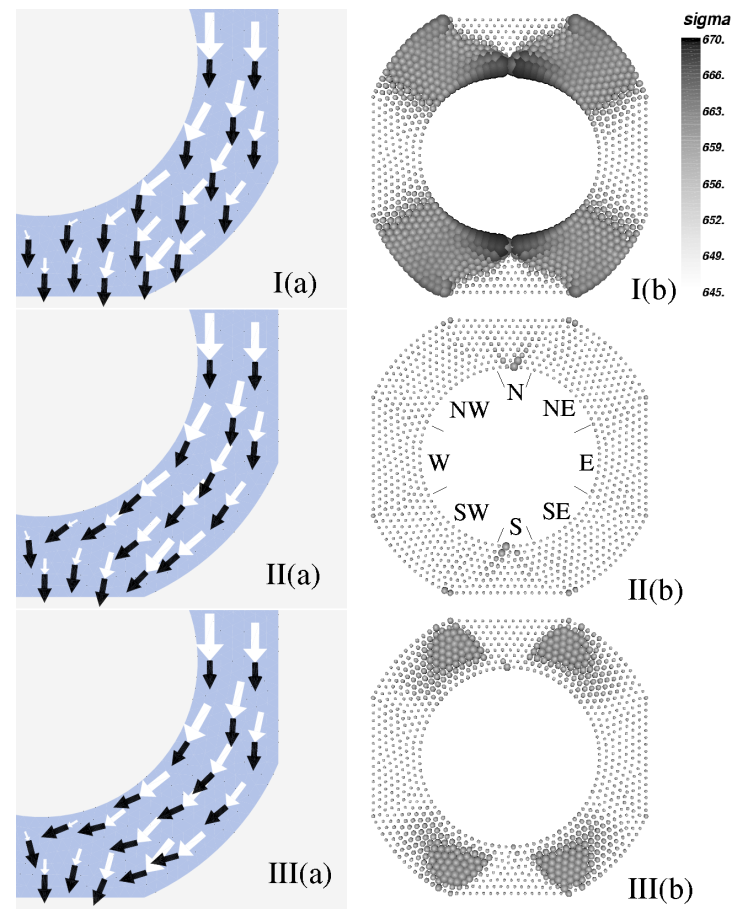

Fig. 3. (a) Magnetization and current density evolution in a section of the ring (with the full picture obtainable by symmetry considerations) and (b) corresponding conductivity distributions for the cases marked in Fig. 2. The magnetization $\mathbf{M}$ is represented by the black arrows, the current density $\mathbf{J}$ by the white ones. The conductivity distribution is shown using bubbles with radius proportional to the local conductivity value $\sigma$. The colour scale, expressed in $\mathrm{S} / \mathrm{m}$, is the same for all plots.

Because of the alignment between the current density and the magnetization the conductivity for point $\mathrm{I}(\mathrm{b})$ is low in the middle section (i.e. East (E) and West (W) from the centre of the ring, see Fig. 3-II (b)) where the magnetization and current density are mostly parallel. The conductivity is large in the diagonal parts (i.e. North-East, North-West, South-West and South-East of the centre of the ring) where there is (roughly) a 45 degree angle between the magnetization and the current density.

At point II, the resistance in Fig. 2(c) reaches the maximum and the applied field is $-18 \mathrm{mT}$. The conductivity (II (b)) is small virtually everywhere throughout the ring in comparison to I (b). This is due to magnetization in the diagonal parts of the ring aligning with the shape of the ring (to reduce the demagnetising energy) and hence with the direction of the current at this applied field.

At point III, the applied field is slightly positive $(22 \mathrm{mT})$ and just below the coercive field $\left(H_{\mathrm{c}}=23 \mathrm{mT}\right)$, and the average magnetization is still pointing downwards in the negative $y$ direction. It can be seen that the magnitude of the $y$-component of the magnetization in the diagonal parts of the ring is smaller than at point II. For this reason there is a larger angle between current density (which has not significantly changed from point I, to II to III) and magnetization, and consequently we see an increased conductivity in these areas.

Finally, we test how important it actually is to compute the spatially varying current density. We compute the conductivity
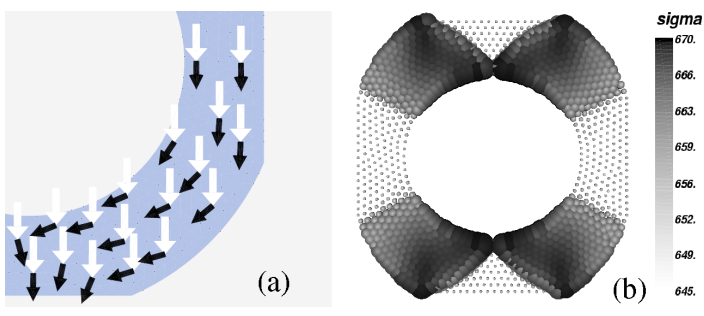

Fig. 4. (a) The magnetization and current density and (b) the conductivity distributions in the uniform current model at the coercive field (point III). The arrows on the edge of the ring in (a) are intended for visualization purposes.

for point III by (wrongly) assuming that the current density would be uniform and would be pointing in the negative $y$-direction everywhere. The results are shown in Fig. 4. The conductivity is significantly overestimated (compare with III (b) in Fig. 3). The AMR ratio obtained from this uniformcurrent calculation is $\approx-4 \cdot 10^{-3}$. Based on the data shown in Fig. 2(c), this corresponds to an applied field of $\approx \pm 200 \mathrm{mT}$ whereas the actual applied field was $22 \mathrm{mT}$.

\section{SUmmary}

We have studied the AMR curve for square arrays of connected rings. Experimental and numerical data agree rather well, and the numerical data allows to intepret the experimental curve. The relation between the AMR and the current distribution is non-linear in the conductivity of the material and we have used a self-consistent approach to determine the AMR values numerically. Finally, we have demonstrated that it is vital to compute the current density correctly to accurately calculate AMR values numerically.

\section{ACKNOWLEDGMENTS}

This work has been funded by the Engineering and Physical Sciences Research Council (EPSRC) in the United Kingdom (GR/T09156/01) and by the National Science Foundation (NSF) in the United States.

\section{REFERENCES}

[1] V. Rose, K. Buchanan, S. H. Chung, M. Grimsditch, V. Metlushko, A. Hoffmann, V. Novosad, S. D. Bader, and H. Ibach, "Frustrated magnetic vortices in a triad of permalloy rings: Magneto-optical kerr effect, magnetic force microscopy, and micromagnetic simulations," Physical Review B, vol. 73, p. 094442, 2006.

[2] M. Kläui, C. A. F. Vaz, L. Lopez-Diaz, J. Rothman, and J. A. C. Bland, "Vortex formation in narrow ferromagnetic rings," Journal of Magnetism and Magnetic Materials, vol. 15, p. R985, 2003.

[3] P. Vavassori, M. Grimsditch, V. Novosad, V. Metlushko, and B. Ilic, "Metastable states during magnetization reversal in square permalloy rings," Physical Review B, vol. 67, no. 13, p. 134429, 2003.

[4] M. Kläui, "Spin switching in mesoscopic ring magnets," Advances in Solid State Physics, vol. 44, p. 479, 2004.

[5] M. J. Donahue and D. G. Porter, OOMMF User's Guide, National Institute of Standards and Technology, Gaithersburg, MD, 1999, interagency Report NISTIR 6376.

[6] S. Blundell, Magnetism in Condensed Matter, 1st ed. Oxford University Press, 2001.

[7] T. R. McGuire and I. Potter, "Anisotropic magnetoresistance in ferromagnetic 3d alloys," IEEE Transactions on Magnetics, vol. 11, no. 4, pp. 1018-1038, 1975.

[8] J. Guo and M. B. A. Jalil, "Transport modelling of py film with antidot array," J. Appl. Phys., vol. 93, no. 10, pp. 7450-7452, 2003.

[9] nmag - a micromagnetic simulation environment, http://www. soton.ac.uk/ fangohr/nsim/nmag. 